\title{
Frontières
}

\section{« Chanter » les morts en pays anyi (Côte-d'Ivoire)}

\section{Véronique Duchesne}

Volume 20, numéro 2, printemps 2008

Les musiques et la mort

URI : https://id.erudit.org/iderudit/018333ar

DOI : https://doi.org/10.7202/018333ar

Aller au sommaire du numéro

Éditeur(s)

Université du Québec à Montréal

ISSN

1180-3479 (imprimé)

1916-0976 (numérique)

Découvrir la revue

Citer cet article

Duchesne, V. (2008). « Chanter » les morts en pays anyi (Côte-d'Ivoire).

Frontières, 20(2), 44-48. https://doi.org/10.7202/018333ar

\section{Résumé de l'article}

Dans le sud-est de la Côte-d'Ivoire, les personnes âgées ont droit à des funérailles solennelles faisant une large place aux manifestations musicales, dansées et chantées. Les lamentations funèbres et le noolo, expressions plutôt chantées, sont exécutées en hommage au défunt. Mais si les lamentations sont plutôt larmoyantes, le noolo est plutôt vigoureux. Et tandis que les paroles des lamentations ont trait à la solitude physique et morale dans laquelle le mort laisse le vivant, au questionnement sur son propre sort, au rôle de médiateur (conféré au défunt) entre le monde des vivants et celui des morts, et finalement renvoient à une certaine impuissance face à la mort, le noolo s'apparente plutôt à un défi lancé à la mort, qui vise à inciter les endeuillés au courage pour vaincre la mort.
Ce document est protégé par la loi sur le droit d'auteur. L'utilisation des services d’Érudit (y compris la reproduction) est assujettie à sa politique d'utilisation que vous pouvez consulter en ligne.

https://apropos.erudit.org/fr/usagers/politique-dutilisation/ 


\section{SURLE $\overline{\text { Noolo exécuté pour les }}$ (O) funérailles de Koffi Brou (C) à Kangandissou dans l'après-midi du 5 décembre 1996. \\ Durée: $7 \min 16 \mathrm{~s}$}

\section{Résumé}

Dans le sud-est de la Côte-d'Ivoire, les personnes âgées ont droit à des funérailles solennelles faisant une large place aux manifestations musicales, dansées et chantées. Les lamentations funèbres et le noolo, expressions plutôt chantées, sont exécutées en hommage au défunt. Mais si les lamentations sont plutôt larmoyantes, le noolo est plutôt vigoureux. Et tandis que les paroles des lamentations ont trait à la solitude physique et morale dans laquelle le mort laisse le vivant, au questionnement sur son propre sort, au rôle de médiateur (conféré au défunt) entre le monde des vivants et celui des morts, et finalement renvoient à une certaine impuissance face à la mort, le noolo s'apparente plutôt à un défi lancé à la mort, qui vise à inciter les endeuillés au courage pour vaincre la mort.

Mots clés: lamentation funèbre chant funéraire - veillée funéraire hommage.

\begin{abstract}
In the south-east of Ivory Coast, the elderly are entitled to solemn funerals with a strong emphasis on musical, danced and sung events. The funeral lament and the noolo, mainly sung expressions, are executed as a tribute to the deceased. But if lamentations are rather tearful, the noolo is rather lively. And while words of lament relate to the physical and moral solitude in which the deceased leaves the living, to the questioning of one's own fate, to the role of mediator (given to the deceased) between the worlds of the living and the dead, and finally refer to a certain helplessness in the face of death, the noolo is more a challenge towards death, which strives to give courage to the mourning in conquering death.
\end{abstract}

Keywords: funeral lament - funeral song - funeral vigil - tribute.

\section{"Chanter » les morts}

\author{
À LA MÉMOIRE DE BROU YAH YINI, \\ DÉCÉDÉE LE 17 AVRIL 2007, À KANGANDISSOU.
}

\section{Véronique Duchesne, Ph.D., \\ membre du Centre d'études des mondes africains (CEMAf), CNRS UMR 8171 EPHE, chargée d'enseignement, Universités Paris I et Paris VIII.}

Dans le sud-est de la Côte-d'Ivoire, en pays any $\mathrm{i}^{1}$, une personne âgée a droit à des funérailles solennelles (êsê kpli, "grandes funérailles »), véritable couronnement dont l'ensemble de sa famille se glorifie. Il arrive aussi que, faute de moyens suffisants dans l'immédiat, la famille décide que le corps du défunt soit gardé à la morgue en attendant une date ultérieurement définie (bê butu êsê, littéralement "on recouvre les funérailles»). La danse ${ }^{2}$ occupe dans le rituel funéraire une place importante. Pendant les sept jours que durent les funérailles, différentes danses peuvent être organisées dans la cour mortuaire, lors des veillées ${ }^{3}$. Chaque danse correspond à un groupe constitué de musiciens, de chanteurs et de danseurs. La danse exécutée varie selon l'âge, le sexe et le statut social du défunt. Ainsi kenyan kpli ${ }^{4}$ estelle exécutée pour les funérailles du roi ou d'un membre du lignage royal. Seuls le roi et la reine sont autorisés à participer à cette danse chaussés et parés d'un couvre-chef, les autres danseurs devant se déchausser et se découvrir. Chaque pas,

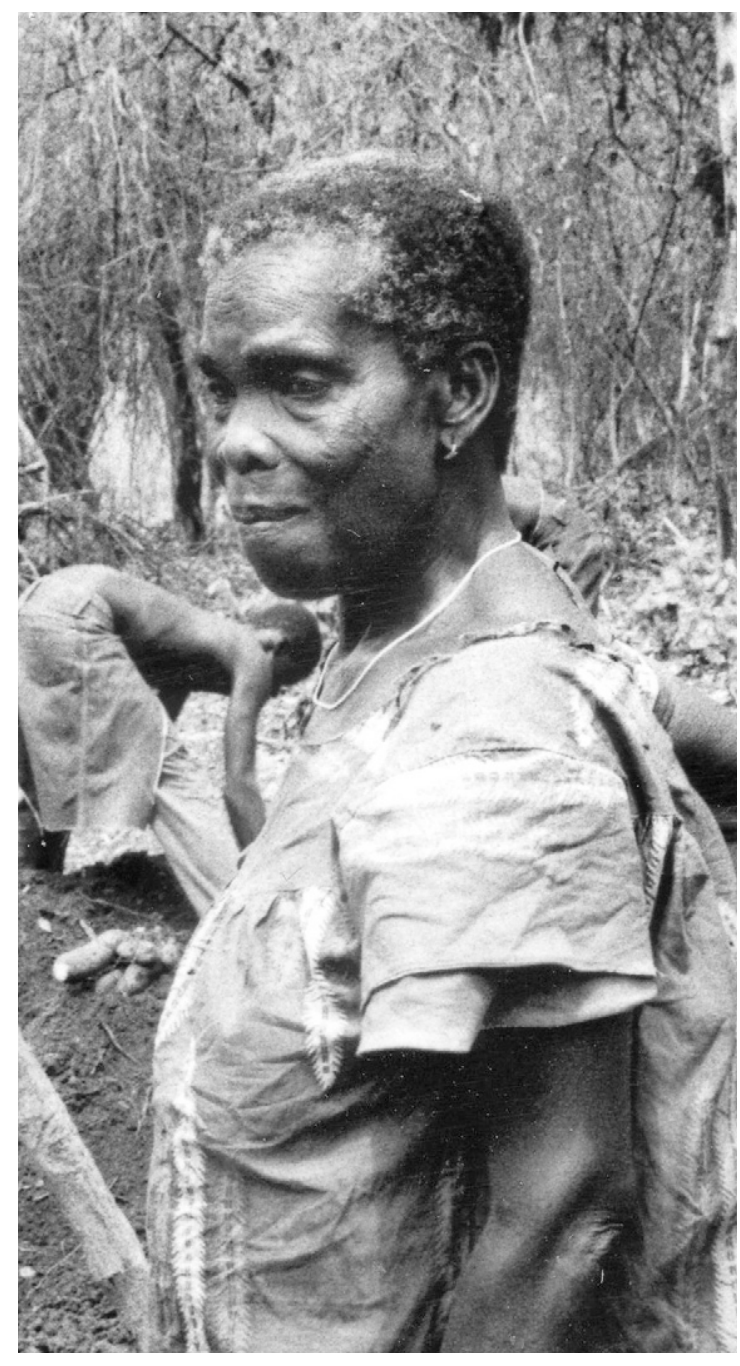

Brou Yah Yini dans son champ d'ignames. 
chaque geste y ont un sens : rien n'y est gratuit. Autrefois, seuls les hommes et femmes libres pouvaient la danser mais, l'esclavage étant aboli, on a tendance à être moins rigoureux. La danse fokwé $e^{5}$ exclusivement masculine est réservée aux hommes appartenant au groupe - aujourd'hui tous âgés. La danse abodan comme la danse kokoubè sont organisées lors de la célébration des funérailles solennelles mais aussi au cours des réjouissances populaires, elles ne comportent pas d'exclusive sociale: hommes, femmes, enfants, vieillards, peuvent en jouir. Alors qu'abodan est dansée par les plus anciens, kokoubè est exécutée par de jeunes gens, en majorité des jeunes filles.

Les funérailles solennelles sont aussi le lieu d'autres expressions sonores (plutôt «chantées» que «dansées») que je souhaite étudier plus particulièrement: les lamentations funèbres et le noolo.

\section{LES LAMENTATIONS FUNÈBRES}

Dans la cour mortuaire, aussitôt l'annonce de la mort et les gestes rituels accomplis (toilette, maquillage avec du kaolin et habillage du défunt), le corps est exposé sur un lit d'apparat, le plus souvent dans le salon qui, pour l'occasion, est décoré de somptueux pagnes tissés aux couleurs chatoyantes. En présence du mort, chacun exprime alors son chagrin d'une façon démonstrative et sonore, en gémissant ou criant, cheveux ébouriffés, mains sur la tête, parfois en se roulant dans la poussière. C'est le temps ritualisé des pleurs (esunê). Hommes, femmes, enfants, participent de cet événement sonore qui retentit jusqu'à l'extrémité du village. Chacun lance ses appels à son gré, appelant le défunt par son nom, et tous les morts de la cour un à un: "Viens secours-moi! Je suis perdu, tu m'as laissé seul. Je suis devenu orphelin. J'ai froid. » Les pleurs constituent un adieu collectif de la communauté à l'un des siens. Ils avertissent également les ancêtres de l'arrivée d'un nouveau membre jouissant de toute l'estime de la communauté.

Passé ce temps des pleurs, les hommes se retirent et s'installent sur des bancs dans la cour et seules les femmes restent auprès du corps. Dans l'espace confiné du salon, le fracas des pleurs a laissé place au silence et à la retenue dans les gestes et les paroles. Soudain une longue complainte s'élève, a cappella: une voix tremblante laisse échapper une tirade de paroles plaintives entrecoupées de sanglots. Cette expression sonore strictement individuelle, dont la structure formelle s'apparente à celle d'un chant monodique (sans accompagnement musical ou dansé) est ce qu'on nomme en français une lamentation funèbre (en anyi esunê bubulê, littéralement «des pleurs cassés $\left.{ }^{6}\right)$. Cette improvisation très per- sonnelle décline les qualités ou les bonnes actions du défunt, sous la forme mélodieuse de louanges adressées au défunt encore présent. Les lamentations ne sont entonnées que par des femmes, le plus souvent âgées ${ }^{7}$, qui connaissent bien le défunt et avec lesquelles ce dernier avait tissé un certain nombre de relations. Les paroles se rapportent toujours au vide que la mort a introduit dans leurs relations personnelles. Elles font référence à un passé commun, proche ou lointain ${ }^{8}$. L'expression-clé autour de laquelle s'organise rythmiquement tout le texte est l'affirmation de la souffrance, omniprésente et éternelle.

\section{Exemple}

À Kangandissou, en novembre 1999, à la mort de sa "fille» [fille de sa sour], une vieille femme entonna la lamentation funèbre suivante:

\section{Ablan, je suis morte}

Tu nous as laissé.

Ablan, que va-t-on devenir sans toi?

La famille est complètement détruite.

Tu nous as laissé.

Tous t'adressent leurs condoléances

(voir Duchesne, 1999, filmographie).

Associant souffrance et éloge, ce «chant» monodique permet d'atténuer la douleur que représente le départ définitif imminent du mort et de consoler à la fois le mort (encore représenté par son corps) et la famille en pleurs. Une autre performance, collective cette fois, remplit aussi cette fonction, mais d'une autre façon.

\section{LE NOOLO}

À la différence des danses présentées précédemment (et qui toutes font référence à un groupe formellement constitué), le noolo ${ }^{9}$ est exécuté par un groupe informel constitué à chaque fois de personnes différentes. Chaque rituel funéraire voit se produire un noolo différent.

Passé le temps des pleurs, des femmes se rassemblent - de rares hommes peuvent également participer - et se munissent de bâtons de bambou coupés dans la longueur. Dès qu'elles sont en nombre suffisant (environ une dizaine), elles forment un cercle et, debout, elles commencent à chanter, chacune frappant l'un contre l'autre deux bâtons (appelé ndê) à un rythme très rapide. Un tambourinaire assis, non loin du cercle, son tambour (appelé kpindrin) entre les genoux, frappe, de ses mains, les rythmes d'accompagnement. À tour de rôle, une chanteuse entonne un bref couplet tandis que les autres lui répondent en chœur. La performance peut durer plusieurs heures. Les solistes se succèdent sans aucune interruption ou tran- sition sonore et dans un ordre tout à fait improvisé. Pour les maintenir en forme, de la boisson leur est offerte par le lignage endeuillé. Quand la performance est terminée, les bâtons utilisés pour rythmer les chants sont jetés à l'entrée du village, ils ne devront plus servir à un autre usage. Le noolo peut être exécuté dès le premier jour des funérailles ou bien être organisé au cours d'une ou plusieurs veillées. En pays anyi, on peut l'entendre indifféremment dans la journée ou dans la nuit ${ }^{10}$.

Précisons que le noolo est aussi exécuté en dehors des funérailles mais avec quelques variantes. Il est alors pratiqué par les petites filles, jeunes filles ou jeunes femmes: disposées en cercle, elles entonnent des chants tout en frappant dans leurs mains. Chaque participante invente son couplet en fonction du refrain choisi par le groupe, ou se contente simplement de chanter un proverbe. Dès qu'elle a fini, elle se laisse tomber, le dos le premier, dans les bras de deux de ses compagnes en prononçant le nom du garçon qu'elle aime ou celui d'un amant fictif. Ses camarades la relèvent en lui faisant faire un saut. C'est là le noolo ordinaire. Celui des funérailles, par contre, est réservé aux adultes et se caractérise par l'absence de la dernière partie de la danse : la chute et le saut pendant qu'on prononce le nom d'un amant. Cela peut toutefois se produire si la défunte est une jeune fille.

Le corpus des chants du noolo est vaste, il ne comporte pas de partie fixe, commune à toutes les funérailles. Tous les chants sont admis, que ce soient les chants de distraction, de fiançailles ou la plainte d'une femme à l'égard de son mari. Pour la circonstance, un couplet évoque souvent le souvenir du disparu ou de celui des membres de sa famille ayant déjà rejoint le séjour des morts. Certains préfèrent improviser des invectives contre la mort: "Mort, si tu étais un homme, je te ferais la guerre », ou évoquer la douleur et l'absence de l'être cher. À part quelques rares couplets réservés au rituel funéraire, les chants du noolo n'ont, par leur nature, aucune relation directe avec le thème de la mort.

Pour ce qui est de la structure formelle, les chants du noolo exécutés collectivement ont une structure responsoriale opposant un soliste à un chœur, dont la forme est donc: ABA'BA'B, etc. [A = soliste, $\mathrm{B}=$ chœur]. S'agissant d'une structure responsoriale, le chœur répète généralement une phrase constante, les variations relevant de la période du soliste. Certains éléments de variations peuvent aussi être intégrés par le chœur. Dans ce cas, la structure peut revêtir la forme suivante: ABA'BA'B' ou toute autre combinaison de ce type. 


\section{Exemple $2^{11}$}

Noolo exécuté pour les funérailles du roi Nanan Nganza II à Kangandissou, dans la nuit du 23 janvier 1991, par Anzera Akouman, Ehoukou Ama, Akissi, Kohuwa, Adjua, Afoua, Adjoba, les chanteuses, et Kadjo et François, les chanteurs, accompagnés par le tambourinaire Yakpo Anzera.

Soliste 1: Moi l'oiseau (bis)

Quel oiseau m'a prédit

qu'un jour je connaîtrai la souffrance.

Chœur: Moi l'oiseau (bis)

Quel oiseau m'a prédit

qu'un jour je connaîtrai la souffrance.

Soliste 2: Je vous remercie

Monsieur Koffi, je vous remercie pour la boisson donnée.

«Madame $»^{12}$ Adjua, je vous remercie

Pour la boisson donnée.

Kouamé oi Kouamé, je te remercie

Pour la boisson donnée.

«Madame» Afouo, pour moi c'est fini.

Chœur: oo oo.

Soliste 3: Il n'y a pas de remède pour celui qui est médisant [on ne peut pas changer celui qui est médisant].

Si ton ennemi est présent quand on juge une affaire,

L'affaire ne finira pas.

Chœur: Il n'y a pas de remède pour celui qui est médisant.

Soliste 4: Boson Ma e [un nom propre]

Mon champ, la forêt.

Chœur: Boson Ma ee.

Soliste 5: «Madame» Eba ee

On sait que tu es quelqu'un de bien.

Chœur: Elle a fait beaucoup d'enfants, ee.

Soliste 5: «Madame» Eba ee

$\mathrm{Si}$ je savais que l'on vient et qu'ensuite on meurt

Je n'allais pas venir.

Chœur: Elle a fait beaucoup d'enfants, ee.

Soliste 5: «Madame» Eba ee

Si je savais que l'on vient et qu'il y a la pauvreté

Je n'allais pas venir.

Chœur: Elle a fait beaucoup

d'enfants, ee.

Seules deux solistes font référence explicitement à la mort. La soliste 2, pour sa part, rend hommage à différentes personnes vivantes en citant leur nom (bê bubu edjo, littéralement «casser le chant»).
Exemple $3^{13}$

Noolo exécuté pour les funérailles de monsieur Koffi Brou à Kangandissou, dans l'après-midi du 5 décembre 1996, par Brou Yah Yini, Brou Ahou, Anzera Akouman, Anzera Etien, Nguetta Ahou, Ehoukou Ama, Ehouman Adjua, Eba Akoua, Kohuwa, Nguessan, les chanteuses, et Akpagni Brou, chanteur, accompagnés par le tambourinaire Brou Kamelan.

Soliste 1 : Ebi Dia, le jour où je verrai mon père, je construirai mon campement toute seule.

Chœur: oo oo, je construirai mon campement toute seule.

Soliste 2: Ebi Dia, le jour où je verrai «madame» Ngô, je construirai mon campement toute seule. [Ngô surnom de Gbalôgô]

Chœur: oo oo, je construirai mon campement toute seule.

Soliste 3: Ebi Dia, le jour où je verrai «madame» Maye, je construirai mon campement toute seule. [Maye est la mère de la soliste] "Madame» Maye dit «Le jour où je verrai monsieur Brou, je construirai mon campement toute seule» [Koffi Brou, le défunt] monsieur Brou dit «Le jour où je verrai madame Yah, je construirai mon campement tout seul» «Madame» Yah dit «Le jour où je verrai madame Ablan, je construirai mon campement toute seule» «Madame» Ablan dit «Le jour où je verrai Gbalôgô, je construirai mon campement toute seule»

Ee Ebi Dia, je suis morte

Chœur: oo oo, je construirai mon campement toute seule.

Soliste 4 : Ebi Dia, le jour où je verrai mon père, je construirai mon campement toute seule.

Chœur: oo oo, je construirai mon campement toute seule.

Soliste 5 : Ebi Dia, le jour où je verrai ma maman, je construirai mon campement toute seule.

Chœur: oo oo, je construirai mon campement toute seule.

Soliste 6: Ebi Dia, le jour où je verrai monsieur Brou, je construirai mon campement toute seule. [le défunt] Monsieur Brou dit «Le jour où je verrai madame Ngô, je construirai mon campement toute seule.» «Madame» Ngô dit «Le jour où je verrai madame Nyanda, je construirai mon campement toute seule.»
«Madame» Nyanda dit «Le jour où je verrai Kakou Amanê, je construirai mon campement

toute seule.»

Kakou Amanê dit «Le jour où je verrai Yao Yah, je construirai mon campement toute seule.»

Yao Yah ee

Chœur: oo oo, je construirai mon campement toute seule.

Soliste 6: Ma sœur, je construirai mon campement toute seule je suis morte, je construis mon campement toute seule.

Chœur: oo oo, je construirai mon campement toute seule ee.

Soliste 6 et soliste 1 : Jouons noolo, soyons énergiques, jouons noolo. Mesdames messieurs, nous jouons noolo.

Chœur: ee ee Jouons noolo.

Soliste 6 et soliste 1: Jouons noolo, Chœur: ee ee Jouons noolo.

Soliste 6: ee jouons noolo, mesdames messieurs, jouons noolo

Chœur: ee ee Jouons noolo.

Soliste 6: Messieurs mesdames, jouons noolo

Chœur: ee ee Jouons noolo.

Soliste 6: Jouons noolo,

Chœur: Jouons noolo, soyons énergiques, jouons noolo

soyons énergiques, jouons noolo.

Soliste 6: Messieurs mesdames, jouons noolo

Mesdames messieurs, jouons noolo

Monsieur Bedia est mort, nous jouons noolo

«Madame» Gbalôgô, je joue noolo [scur décédée de la soliste]

«Madame» Anzera Nyanda, je m'amuse [soeur décédée de la soliste]

Yao Yah, je m'amuse

Koffi Brou, je m'amuse [le défunt].

Chœur: Jouons noolo, soyons énergiques, jouons noolo soyons énergiques, jouons noolo.

Soliste 7 : Gbaniye, je suis morte (ter). Ce que tu feras en chemin, on le saura Où tu dormiras, on le saura.

e Asafo ee (bis)

Je n'irai pas là-bas.

Monsieur Houphoüet, ne te fâche pas [feu Houphoüet Boigny, président de la République de Côte-d'Ivoire]. 


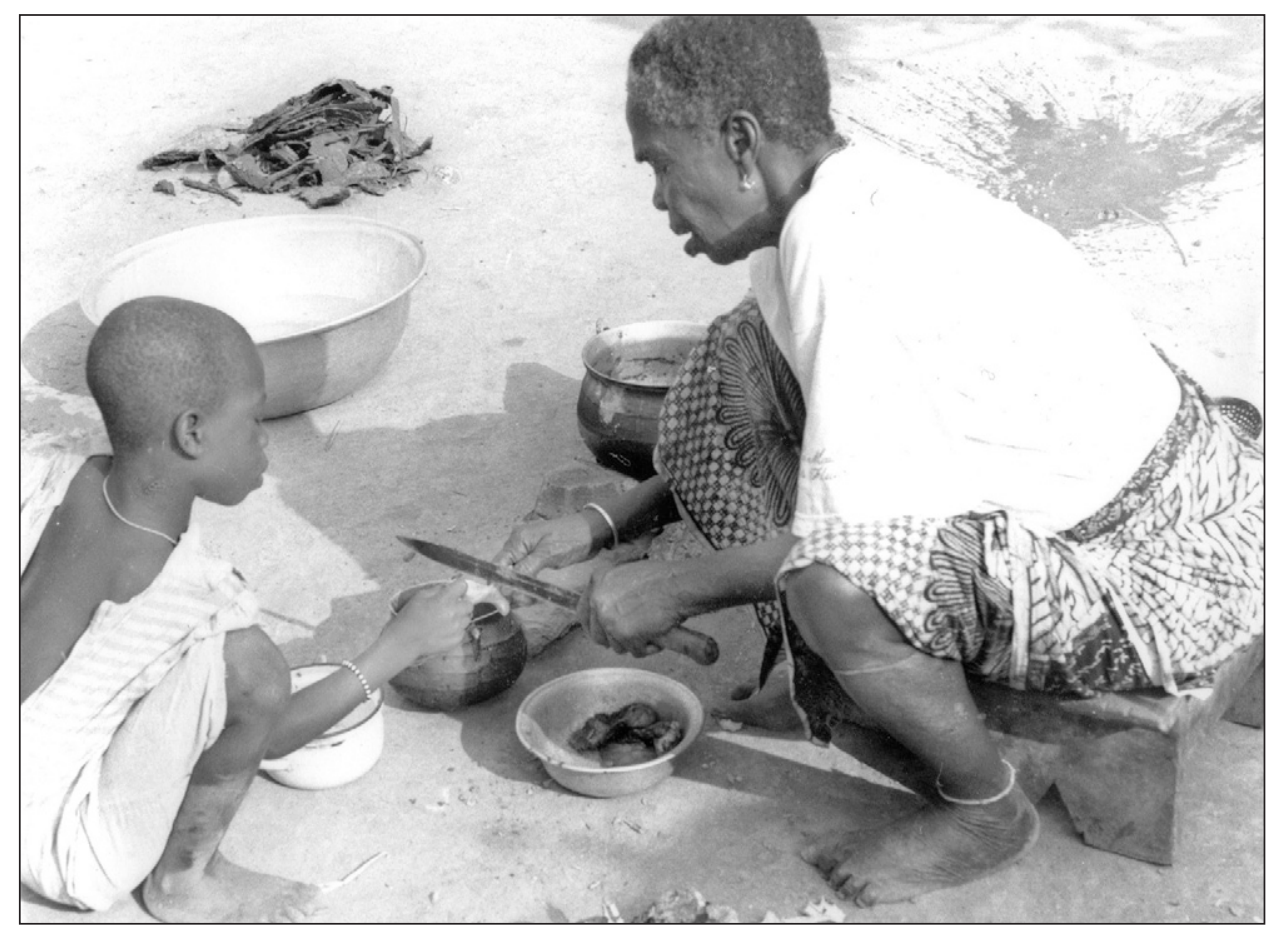

Brou Yah Yini prépare le repas de la veuve (funérailles de Koffi Brou, Kangandissou, le 5 décembre 1996).

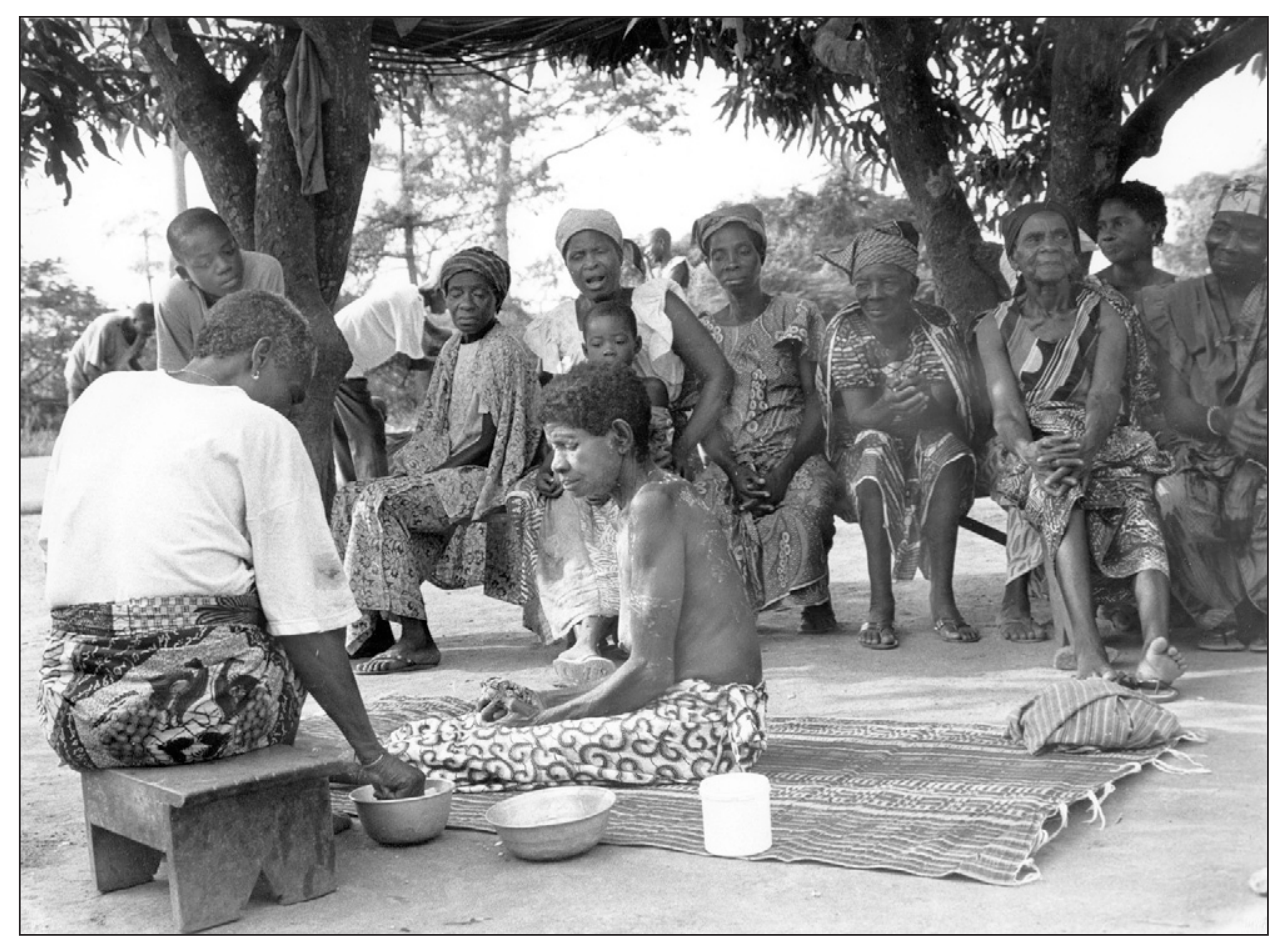

Repas rituel de la veuve (funérailles de Koffi Brou, Kangandissou, le 5 décembre 1996).

Tous ceux qui font du mal, montre-leur qui tu es.

Anungble Anungble deux queues d'animal jumelles

Chœur: Anungble Anungble.

Soliste 6: Papa je suis morte (bis).

Je suis là, le problème de cette année

Si on n'arrive pas à le régler

Il faut le confier aux militaires. d'animal jumelles

Chœur: Anungble

Soliste 6: Notre village.

Chœur: Anungble.

Soliste 6: J'ai fait jusqu'à je n'ai pas fait d'enfants.

Chœur: Anungble.

Soliste 6: Notre village.
Anungble Anungble, deux queues
Chœur: Anungble.

Soliste 5 : Notre village.

Chœur: Anungble.

Soliste 6: ee Ana ee ee (bis)

Chœur: a a.

Soliste 7: Asafue, je vous remercie.

Chœur: ee

Soliste 7 : On vous remercie.

Chœur: an an.

Soliste 7 : Koffi Brou dit qu'il vous remercie [le défunt].

Chœur: ee.

Soliste 8: Avant je ne savais pas.

Maintenant j'ai compris.

Chœur: Maintenant j'ai compris,

Avant je ne savais pas.

Amorcer un noolo constitue une réponse immédiate et énergique des proches (parents, alliés, amis) qui refusent que l'abattement moral et physique envahisse les deuilleurs ${ }^{14}$. L'orchestre du noolo est d'ailleurs facilement constitué puisqu'il comprend des idiophones (les bâtons) dont la fabrication n'est pas difficile et un membranophone (un tambour à une membrane) couramment utilisé non seulement dans les rituels funéraires mais également dans les rites pour les jumeaux ou pour les cérémonies de divination publique.

Les lamentations funèbres et le noolo ont pour caractéristique commune de faire une large place à l'improvisation: on ne sait pas à l'avance qui va entonner une lamentation ou bien qui va exécuter un noolo. Dans les deux cas, en réaction à l'émotion liée à la disparition d'un proche, des individus choisissent de s'exprimer à travers ces manifestions plus ou moins chantées. Mais si les lamentations sont plutôt larmoyantes, le noolo est plutôt vigoureux (leur tempo diffère d'ailleurs complètement: très lent et très rapide). Et tandis que les paroles des lamentations ont trait à la solitude physique et morale dans laquelle le mort laisse le vivant, au questionnement sur son propre sort, au rôle de médiateur (conféré au défunt) entre le monde des vivants et celui des morts, et finalement renvoient à une certaine impuissance face à la mort, le noolo s'apparente plutôt à un défi lancé à la mort, qui vise à inciter les deuilleurs au courage pour vaincre la mort. Tandis que les lamentations exécutées dans l'intimité du salon en présence du défunt expriment l'antithèse de la vie, le noolo exécuté à l'extérieur, devant les deuilleurs, avant l'enterrement ou pendant les veillées, exprime la vie sous un angle plus clément.

Par conséquent, il ne faut pas s'étonner de l'apparition récente, au cours des 


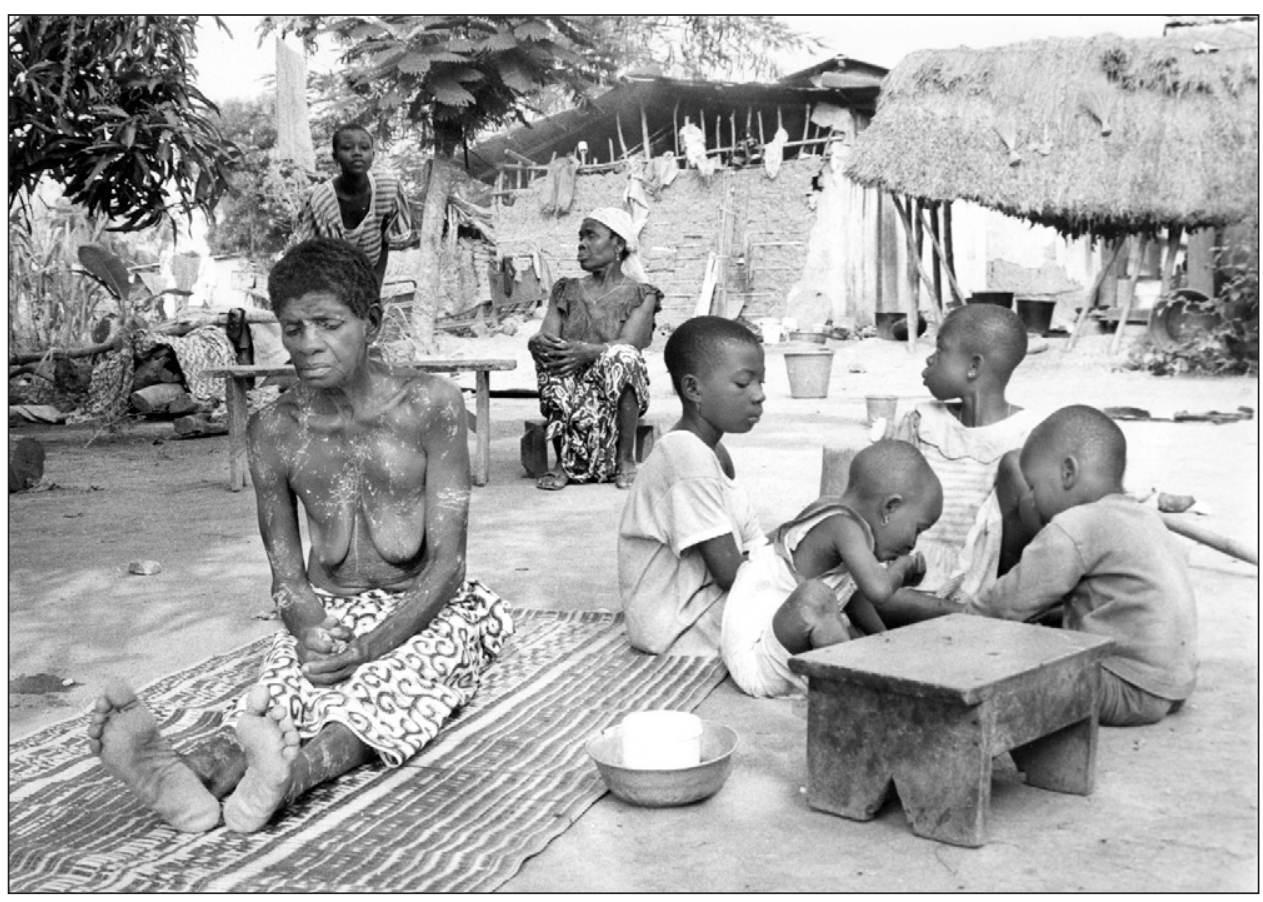

Les enfants mangent les restes du repas de la veuve (funérailles de Koffi Brou, Kangandissou, le 5 décembre 1996).

funérailles anyi, de nouvelles expressions festives du triomphe de la vie sur la mort, dans la continuité du noolo. Beaucoup de veillées sont aujourd'hui animées par un DJ qui fait écouter les cassettes des artistes régionaux et nationaux, musiques sur lesquelles dansent surtout les plus jeunes. Par ailleurs, lorsque le défunt est de confession chrétienne, la chorale de l'Église (catholique ou protestante) tient désormais une place importante au cours de ces veillées religieuses. Enfin, expressions du statut social du défunt mais aussi de la situation économique de sa famille, l'animation musicale - tout comme la nourriture et la boisson - est plus que jamais essentielle lors de ces cérémonies: certaines veillées funéraires qui souvent ont lieu le samedi soir sont ainsi appelées «Tonnerre »nom d'une émission de variétés télévisée ivoirienne très populaire - parce qu'on est certain de bien s'amuser toute la nuit tandis que celles qui sont organisées par une famille pauvre sont qualifiées de «Bal poussière ». Si la célébration des funérailles évolue rapidement en pays anyi ${ }^{15}$, ces changements, en définitive, concernent, davantage la forme que l'esprit!

\section{Bibliographie}

DUCHESNE, V. (1996). Le cercle de kaolin. Boson et initiés en Côte d'Ivoire, Paris, Institut d'ethnologie.

ESCHLIMANN, J. P. (1988). Les Agni devant la mort, Paris, Karthala.

GUERRY, V. (1970). La vie quotidienne dans un village baoulé, Abidjan, INADES.

NKETIA, J. H. Kwabena (1969). Funeral Dirge of the Akan People, New York, Negro University Press.
OPOKU, A. M. (1970). "The dance in Traditional African Society», Research Review, vol. 7, $\mathrm{n}^{\circ} 1$.

OWUSU-SARPONG, C. (2000). La mort akan. Étude ethno-sémiotique des textes funéraires akan, Paris, L'Harmattan.

PERROT, C.-H. (1982). Les Anyi-Ndényé et le pouvoir aux XVIII et XIXXe siècles, Abidjan Paris, Ceda - Publications de la Sorbonne.

SAHIRI, Léandre (2004). Les obsèques de Bahi Oromé, Abidjan, Kasimex.

\section{Filmographie}

DUCHESNE, V. (1999). Funérailles d'Ablan Yôbouô, DV Cam, Dépôt Base de données Santé-Maladie-Malheur, Paris, Muséum national d'histoire naturelle, SMM.

\section{Notes}

1. La Côte-d'Ivoire compte plus de 400000 locuteurs anyi. Se reconnaissent réciproquement être des Anyi: les Sanwi, les Morofouè, les Ndényé, les Diabé ou Djouabli, les Bini et les Bona. Depuis 1990, je mène des enquêtes ethnographiques auprès des Anyi Morofouè dans le département de Bongouanou. Ce sont mes photos qui accompagnent le texte.

2. Dans le contexte anyi - et plus généralement cher à distinguer danse, chant et musique et à les définir abstraitement: en effet un même nom propre sert à identifier à la fois un répertoire de chants, un orchestre (un ensemble d'instruments) et des pas de danse. Pourtant les performances mentionnées ci-après sont bien désignées par le même terme anyi bê si, traduit en français par «danser»: on dit be si kenyan kpli pour "danser kenyan kpli», bê si fokwe pour "danser fokwe », bê si abodan pour «danser abodan», bê si kokoubè pour «danser kokoubè». dans le contexte africain -, il est vain de cher-
3. Voir le site Internet $<$ http ://www.afritheatre. com/index.asp? navig=fiche\&no_piece $=31>$ où un auteur de théâtre ivoirien, François Amon d'Aby, traite de ce sujet dans sa pièce : Kwao Adjoba.

4. Kenyan kpli qui signifie "grand tambour», désigne à la fois l'instrument de musique et la danse qu'il accompagne.

5. Fokwe désigne à la fois le long tambour frappé à l'aide de deux bâtons recourbés, la danse et le groupe constitué.

6. Les Anyi Bona emploient l'expression bê yi ayilê, "on parle tout en pleurant» (Eschlimann, 1985, p. 157, note 6).

7. Eschlimann, qui a travaillé en pays anyi-bona, précise que: "[La lamentation funèbre] ne peut être exécutée que par les vieilles femmes ou par celles qui ont fait au moins six enfants. Les jeunes filles ne peuvent s'y mêler. On craint que la stérilité et la mort les frappent si elles y participent et que, vu leur jeune âge, elles ne soient tentées de se servir de la lamentation comme d'un amusement» (1985, p. 157).

8. Ce que mentionne Perrot, qui a étudié l'histoire des Anyi-Ndényé: «De même que le mort, dont le corps est étendu sur le lit d'apparat, entr'ouvre la barrière qui sépare les vivants des morts et parvient chez ceux-ci chargé des messages que leur confient, au nom de leur proches, les pleureuses, de même les lamentations des femmes font revivre pour les assistants un passé proche ou lointain» (1982, p. 7-8).

9. Alors que certains auteurs - comme Eschlimann (1985) et Guerry (1970, p. 62-63) - qualifient le noolo de "danse», je dois préciser que l'expression anyi utilisée est bê bo noolo traduite par «jouer noolo" (ou «frapper noolo»), l'expression bê si noolo, «danser noolo » n'est jamais utilisée. Le même verbe (bê bo) est par ailleurs utilisé pour bê bo kenyan, "jouer du tambour» ou pour bê bo njuru, "jouer du xylophone», ainsi que pour bê bo aosi, «jouer aosi », où aosi est un groupe constitué d'un orchestre spécifique et partageant un même répertoire de chants.

10. Selon Guerry (1970, p. 62), en pays voisin baoulé, la «danse funèbre » - il s'agit du noolo - «ne doit jamais être commencée en plein jour».

11. Je remercie Koffi Kouadio pour son aide précieuse dans la traduction des paroles des chants présentés ci-après.

12. Traduction du mot anyi Mo qui est une marque de respect envers une femme.

13. Reproduit sur le CD accompagnant ce numéro de Frontières.

14. Un vieil homme baoulé explique à Vincent Guerry, étonné par l'ambiance joyeuse que manifeste le noolo: «Nous sommes tous dans la tristesse et nous souffrons beaucoup de la mort de notre frère. Mais il n'est pas bon que la famille du défunt reste seule toute la nuit à pleurer, il faut les distraire de leur chagrin ; c'est pourquoi nous jouons, nous dansons devant eux » (1970, p. 63).

15. L'écrivain ivoirien Léandre Sahiri aborde le sujet de l'évolution récente des cérémonies funéraires, qui dépasse le seul contexte anyi, dans son dernier roman: Les obsèques de Bahi Oromé (2004). 